\title{
HOMEOMORPHISM GROUPS OF SOME DIRECT LIMT SPACES
}

\author{
MARGIE HALE ${ }^{1}$
}

\begin{abstract}
Let $F$ be either of the spaces $R^{\infty}=\underline{\lim } R^{n}$ or $Q^{\infty}=\underline{\lim } Q^{n}$ where $R$ denotes the reals and $Q$ the Hilbert cube. Let $\mathcal{H}(M)$ be the homeomorphism group of a connected $F$-manifold $M$ with the compact-open topology. We prove that $\mathcal{H}(M)$ is separable, Lindelöf, paracompact, non-first-countable, and not a $k$-space.
\end{abstract}

Let $R$ denote the reals and $Q$ the Hilbert cube. Let $F$ denote either of $R^{\infty}=$ $\stackrel{\lim }{\longrightarrow} R^{n}$ or $Q^{\infty}=\underline{\lim } Q^{n}$. It is known that $F$ is paracompact [7, III.1] but not first countable [6, p. 391], hence not metrizable. By an $F$-manifold we mean a paracompact space which is locally homeomorphic to $F$.

Work by Heisey and Liem shows that the behavior of $F$-manifolds is similar to that of metrizable, infinite dimensional manifolds. Let $l_{2}$ denote separable, infinite dimensional Hilbert space. Just as for $l_{2}$-manifolds, $F$-manifolds are triangulable, stable on multiplication by the model, and classified by homotopy type; also, each connected $F$-manifold embeds as an open subset of $F$ (see Heisey [8 and 9]). Recently, $\alpha$-approximation theorems $[10,12]$ and unknotting theorems $[11,12]$ have been achieved for $F$-manifolds, similar to those for $Q$-manifolds.

For any space $X$, let $\mathcal{H}(X)$ denote the space of all homeomorphisms of $X$ onto itself with the compact-open topology. $H(X)$ is a group under composition of functions, though not necessarily a topological group. In the metrizable case, Renz [13] has shown that $H\left(l_{2}\right)$ and $H(Q)$ are contractible. (Recall that $l_{2} \cong \prod_{1}^{\infty} R[1]$.) Further, a theorem completed by Ferry [3] states that the homeomorphism group of a compact $Q$-manifold is an $l_{2}$-manifold.

The problem we address here is the nature of the homeomorphism group of an $F$-manifold $M$. In [6] Heisey proved that $\mathcal{H}(F)$ is contractible. Previous work [4] by the author shows that $\mathcal{H}(M)$ is $F$-stable; that is, $H(M) \times F \cong H(M)$. As noted above, stability is a property shared by $F$-manifolds. In this paper we show that, also like $M, \mathcal{H}(M)$ is separable, Lindelöf, paracompact, and non-first-countable. However, we also show that $\mathcal{H}(M)$ is not a $k$-space, and hence not an $F$-manifold.

The author would like to thank her thesis advisor, Richard Heisey, for his advice and support during the course of this work.

Call a space $X$ weakly second countable if there is a countable collection $D=$ $\left\{D_{k} \mid k=1,2, \ldots\right\}$ of subsets of $X$, not necessarily open, such that for each $x \in X$ and each open set $U$ of $X$ containing $x$ there is an integer $k$ such that $x \in D_{k} \subset U$.

Received by the editors July 6, 1981 .

1980 Mathematics Subject Classification. Primary 57S05.

Key words and phrases. Homeomorphism group, direct limit, compact-open topology, manifold.

${ }^{1}$ This research was done while the author was supported by a Susan B. Riley Fellowship from the American Association of University Women, and constitutes part of the author's doctoral dissertation at Vanderbilt University.

(c) 1982 American Mathematical Society 0002-9939/81/0000-0395/802.25 
Clearly each second countable space is weakly second countable, as well as any countable union of second countable spaces. Thus, $F$ is weakly second countable, but not second countable.

Proposition 1. A weakly second countable space is separable and Lindelöf.

The proof of Proposition 1 follows the proofs for second countability [2, VIII, $6.3,7.3]$.

A space $X$ is a countable direct limit of compact metric spaces (CDLCMS) if $X=\lim _{\longrightarrow} C_{n}$, where each $C_{n}$ is a compact metric subspace of $C_{n+1}$. Examples are $R^{n}, F$, and any connected $F$-manifold [7, III.2]. Since a CDLCMS is Hausdorff and regular $[5,4.1,4.3]$ we have

PROPOSITION 2 [5, 1.1]. If $X$ is a CDLCMS, then $\mathcal{H}(X)$ is Hausdorff and regular.

NotAtion. The identity map on a space $X$ will be denoted by id $X$. If $A, B \subset$ $X$, then $(A, B)=\{h \in \mathcal{H}(X) \mid h(A) \subset B\}$. The closure of $A$ in $X$ is written $\operatorname{cl}_{X}(A)$.

THEOREM 1. If $X$ is a CDLCMS, then $H(X)$ is weakly second countable.

THEOREM 2. If $X$ is a CDLCMS, then $\mathscr{H}(X)$ is separable, Lindelöf, and paracompact.

REMARK. Theorems 1 and 2 apply to spaces $X$ which are connected $F$ manifolds, as noted above.

Theorem 2 follows easily from Theorem 1 by applying Propositions 1 and 2 and [2, VII, 6.5].

Proof OF THEOREM 1. Write $X=\varliminf_{n} C_{n}$ as in the definition of CDLCMS. Choose a countable collection $D=\left\{D_{k} \mid k=1,2, \ldots\right\}$ of subsets of $X$ satisfying

(i) Each $D_{k}$ is a compact neighborhood in some $C_{n}$;

(ii) $D$ contains a (compact) basis for each $C_{n}$.

Let $f \in(K, W) \subset \mathcal{H}(X)$, where $K$ is compact and $W$ is open. (Thus $(K, W)$ is a typical subbasic open set in $\mathcal{H}(X)$.) By $[5,2.4]$ there are integers $m, n$ such that $K \subset C_{n}$ and $f\left(C_{n}\right) \subset C_{m}$. Now, $K \subset f^{-1}(W) \cap C_{n}$, which is open in $C_{n}$, so there is a finite set $M$ of integers such that

$$
K \subset \bigcup_{k \in M} D_{k} \subset f^{-1}(W) \cap C_{n}
$$

By similar reasoning, there is a finite set $N$ of integers such that

$$
f\left(\bigcup_{k \in M} D_{k}\right) \subset \bigcup_{l \in N} D_{l} \subset W \cap C_{m} .
$$

Thus, $f \in\left(\bigcup_{k \in M} D_{k}, \bigcup_{l \in N} D_{l}\right) \subset(K, W)$.

Now, let $\mathcal{U} \subset \mathcal{H}(X)$ be an arbitrary open set containing $f$. Then there is some basic open set $O=\bigcap_{i=1}^{j}\left(K_{i}, W_{i}\right)$ such that $f \in O \subset \mathcal{U}$. As above, for each $i$, there are finite sets $M_{i}$ and $N_{i}$ of integers such that

$$
f \in \bigcap_{i=1}^{j}\left(\bigcup_{k \in M_{i}} D_{k}, \bigcup_{l \in N_{i}} D_{l}\right) \subset 0 \subset U .
$$

But the collection of all possible sets of this form is countable, and the theorem is proved. 
We now turn to two further theorems. As before, $M$ denotes an $F$-manifold (not necessarily connected).

THEOREM 3. $\mathcal{H}(M)$ is not first countable.

THEOREM 4. $\mathcal{H}(M)$ is not a $k$-space.

Note that $R^{\infty}$ is a factor of $M[9$, Theorem 1,8 , Theorem 7]. We prove Theorems 3 and 4 for the special case $M=R^{\infty}$ (Theorems 5 and 6 below). The general case then follows from the following

Proposition 3. For Hausdorff spaces $X$ and $Y$, the map $\phi: \mathcal{H}(X) \rightarrow \mathcal{H}(X \times Y)$ defined by $\phi(h)=h \times \mathrm{id}_{Y}$ is a closed embedding.

The proof of Proposition 3 is routine and will be omitted.

THEOREM 5. $\nVdash\left(R^{\infty}\right)$ is not first countable.

ProOF. Let $C=\left\{\left(K_{i}, W_{i}\right) \mid i=1,2, \ldots\right\}$ be any countable collection of subbasic neighborhoods of $\operatorname{id}_{R^{\infty}}$ in $\mathcal{H}\left(R^{\infty}\right)$. It will suffice to show that there is a neighborhood $U$ of $0=(0,0, \ldots) \in R^{\infty}$ such that no finite intersection $\bigcap_{i=1}^{n}\left(K_{i}, W_{i}\right)$ is contained in $(\{0\}, U)$.

Since $R^{\infty}$ is separable and locally path-connected, the collection $P$ of all path components of all sets of the form

$$
\begin{aligned}
& R^{\infty}-\left(\bigcup_{k=1}^{r} K_{i(k)}\right) ; \text { or } \\
& \bigcap_{j=1}^{m} W_{i(j)} ; \text { or } \\
& \left(\bigcap_{j=1}^{m} W_{i(j)}\right)-\left(\bigcup_{k=1}^{r} K_{i(k)}\right),
\end{aligned}
$$

where $m$ and $r$ take on all integer values, is countable. Since $R^{\infty}$ is not first countable, $P$ does not form a basis for $R^{\infty}$ at 0 , and hence there is a neighborhood $U$ of 0 such that if $0 \in P$ and $P \in P$, then $P \not \subset U$.

Let $n$ be given. We must show that $\bigcap_{i=1}^{n}\left(K_{i}, W_{i}\right) \not \subset(\{0\}, U)$. The argument breaks down into three cases, all handled similarly.

Case (i): $0 \notin \bigcup_{i=1}^{n} K_{i}$. Set $V=R^{\infty}-\left(\bigcup_{i=1}^{n} K_{i}\right)$. Let $P$ be the path component of $V$ containing 0 . So $P \in P$ and there is a point $y \in P-U$. The map taking 0 to $y$ is an embedding into $R^{\infty}$ homotopic to inclusion. Using Lemma 3.1 of [12] we extend to a homeomorphism $h$ of $R^{\infty}$, fixed outside $P$, and taking 0 to $y$. Then $h \in\left[\bigcap_{i=1}^{n}\left(K_{i}, W_{i}\right)\right]-(\{0\}, U)$.

Case (ii): $0 \in \bigcap_{i=1}^{n} K_{i}$. Set $V=\bigcap_{i=1}^{n} W_{i}$ and repeat the arguments of Case (i). Case (iii): $0 \in\left(\bigcap_{i=1}^{m} K_{i}\right)-\left(\bigcup_{i=m+1}^{n} K_{i}\right), 1 \leq m<n$. Set $V=\left(\bigcap_{i=1}^{m} W_{i}\right)-$ $\left(\bigcup_{i=m+1}^{n} K_{i}\right)$ and repeat the preceding argument.

Thus the proof is complete.

THEOREM 6. $\mathcal{H}\left(R^{\infty}\right)$ is not a $k$-space.

ProOF. Consider $R^{\infty}$ as a topological vector space with basis $B=\left\{e_{n} \mid n=\right.$ $1,2, \ldots\}$, where $e_{n}=(0, \ldots, 0,1,0, \ldots), 1$ in the $n$th component. Let $\mathcal{L}\left(R^{\infty}\right)$ 
denote the space of all linear maps of $R^{\infty}$ into itself, with the compact-open topology. Define $\mathcal{L} H\left(R^{\infty}\right)=\mathcal{L}\left(R^{\infty}\right) \cap \mathcal{H}\left(R^{\infty}\right)$.

It is easy to see that the map $\psi: \mathcal{L}\left(R^{\infty}\right) \rightarrow \Pi_{1}^{\infty} R^{\infty}$ defined by $\psi(f)=\left(f\left(e_{1}\right)\right.$, $\left.f\left(e_{2}\right), \ldots\right)$ is a homeomorphism taking $\mathcal{L} H\left(R^{\infty}\right)$ onto the subset

$$
Y=\left\{\left(x_{1}, x_{2}, \ldots\right) \mid\left\{x_{1}, x_{2}, \ldots\right\} \subset R^{\infty} \text { is a basis }\right\}
$$

Now, $\mathcal{L} H\left(R^{\infty}\right)$ is a closed subspace of $H\left(R^{\infty}\right)$, so in order to prove Theorem 6 it suffices to prove that $\mathcal{L} \mathcal{H}\left(R^{\infty}\right)$, or $Y$, is not a $k$-space.

We construct a set $A$ in $Y$ whose intersection with every compact set is closed, but which is not itself closed. This construction is similar to [6, II-1(b)].

Let $e=\left(e_{1}, e_{2}, \ldots\right) \in Y$. For positive integers $r$ and $j$, define

$$
x_{r}^{j}=\frac{1}{r} \cdot e_{j}=\left(0, \ldots, 0, \frac{1}{r}, 0,0, \ldots\right) \in R^{\infty} .
$$

For $j \geq 2$, define $A_{j} \subset Y$ by

$$
A_{j}=\left\{\left(e_{1}+x_{r}^{j}, e_{2}, \ldots, e_{j-1}, e_{j}+e_{k}, e_{j+1}, \ldots\right) \in \prod_{1}^{\infty} R^{\infty} \mid k \geq 2, r \leq k\right\} .
$$

We show that indeed $A_{j} \subset Y$. Let $j, k \geq 2$ and $r \leq k$ be given. Of the elements

$$
e_{1}+x_{r}^{j}, e_{2}, \ldots, e_{j-1}, e_{j}+e_{k}, e_{j+1}, \ldots,
$$

for $m \notin\{1, j, k\}, e_{m}$ is the only one whose $m$ th component is nonzero. Thus it suffices to show that the remaining elements form a linearly independent set, and that all the elements span $R^{\infty}$.

Case (i): $j \neq k$. Assume

$$
\begin{aligned}
0 & =\lambda_{1}\left(e_{1}+x_{r}^{j}\right)+\lambda_{2}\left(e_{j}+e_{k}\right)+\lambda_{3} e_{k} \\
& =\left(\lambda_{1}, 0, \ldots, 0, \lambda_{2}+\lambda_{1} / r, 0, \ldots, 0, \lambda_{2}+\lambda_{3}, 0, \ldots\right) .
\end{aligned}
$$

Then $\lambda_{i}=0$ for $1 \leq i \leq 3$, and the set is linearly independent.

Let $x=\left(x_{1}, x_{2}, \ldots\right)=\sum_{i=1}^{\infty} x_{i} e_{i} \in R^{\infty}$. It is routine to verify that

$$
x=x_{1}\left(e_{1}+x_{r}^{j}\right)+\left(x_{j}-\frac{x_{1}}{r}\right)\left(e_{j}+e_{k}\right)+\left(x_{k}-x_{j}+\frac{x_{1}}{r}\right) e_{k}+\sum_{i \neq 1, j, k} x_{i} e_{i} .
$$

Case (ii): $j=k$. If $0=\lambda_{1}\left(e_{1}+x_{r}^{j}\right)+\lambda_{2}\left(2 e_{j}\right)=\left(\lambda_{1}, 0, \ldots, 0, \lambda_{1} / r+2 \lambda_{2}, 0, \ldots\right)$, then $\lambda_{1}=0=\lambda_{2}$, so the set is linearly independent.

Again, one can easily check that

$$
\sum_{i=1}^{\infty} x_{i} e_{i}=x_{1}\left(e_{1}+x_{r}^{j}\right)+\frac{1}{2}\left(x_{j}-\frac{x_{1}}{r}\right)\left(2 e_{j}\right)+\sum_{i \neq 1, j} x_{i} e_{i} .
$$

Thus each $A_{j} \subset Y$ and we define $A=\bigcup_{j=2}^{\infty} A_{j} \subset Y$. This is the set we desired. If $C \subset Y$ is compact, then $C \subset \prod_{i=1}^{\infty} R^{n_{i}}$, for some integers $n_{i}[5,2.4]$. We show that $A \cap\left(\prod_{1}^{\infty} R^{n_{i}}\right)$ is empty or finite. Thus $A \cap C$ will be empty or finite, and hence closed in $C$.

For a given integer $j$, if $A_{j}$ meets $\prod_{1}^{\infty} R^{n_{i}}$, then $e_{1}+x_{r}^{j} \in R^{n_{1}}$, for some $r$. Hence $j \leq n_{1}$, and only finitely many of the sets $A_{j}$ meet $\prod_{1}^{\infty} R^{n_{i}}$. Fix $j$ between 
2 and $n_{1}$ and suppose

$$
\left(e_{1}+x_{r}^{j}, e_{2}, \ldots, e_{j-1}, e_{j}+e_{k}, e_{j+1}, \ldots\right) \in A_{j} \cap\left(\prod_{1}^{\infty} R^{n_{i}}\right) .
$$

Then $e_{j}+e_{k} \in R^{n_{j}}$, so $k \leq n_{j}$, and there are only finitely many choices for $k$. By definition of $A_{j}, r \leq k$, so there are only finitely many choices for $r$. That is, $A_{j} \cap\left(\Pi_{1}^{\infty} R^{n_{i}}\right)$ is finite.

It remains to show that $A$ is not closed in $Y$. We show that $e \in \operatorname{cl}_{Y}(A)-A$. Clearly $e \notin A$, since $x_{r}^{j}$ is never 0 . Choose a basic neighborhood of $e$ in $Y$. We may assume this neighborhood is of the form $V=\left[\prod_{i=1}^{n}\left(e_{i}+U\right) \times \prod_{n+1}^{\infty} R^{\infty}\right] \cap Y$, where $U=\left(\prod_{l=1}^{\infty} U_{l}\right) \cap R^{\infty}$ and $U_{l}$ is a neighborhood of 0 in $R[6, \Pi-1(\mathrm{a})]$. Choose $r \geq 2$ such that $1 / r \in U_{n+1}$. Then the point

$$
\left(e_{1}+x_{r}^{n+1}, e_{2}, \ldots, e_{n}, e_{n+1}+e_{r}, e_{n+2}, \ldots\right)
$$

is in $A_{n+1} \cap V$. So $e \in \operatorname{cl}_{Y}(A)$.

Thus Theorem 6 is proved.

\section{REFERENCES}

1. R. D. Anderson, Hilbert space is homeomorphic to the countable infinite product of lines, Bull. Amer. Math. Soc. 72 (1966), 515-519.

2. James Dugundji, Topology, Allyn and Bacon, Boston, Mass., 1966.

3. Steve Ferry, The homeomorphism group of a compact Hilbert cube manifold is an ANR, Ann. of Math. (2) 106 (1977), 101-119.

4. Margie Hale, A factoring technique for homeomorphism groups (to appear).

5. V. L. Hansen, Some theorems on direct limits of expanding sequences of manifolds, Math. Scand. 29 (1971), 5-36.

6. R. E. Heisey, Contracting spaces of maps on the countable direct limit of a space, Trans. Amer. Math. Soc. 193 (1974), 389-411.

7. (1975), 295-312.

8. __ Manifolds modeled on the direct limit of Hilbert cubes, Proc. Conf. Geometric Topology, Univ. of Georgia, 197.7.

9. - Stability, classification, open embeddings, and triangulation of $R^{\infty}$-manifolds, Proc. Internat. Conf. Geometric Topology, Polish Scientific Publishers, Warsaw, 1980, pp. 193-196.

10. Vo-Thanh Liem, An $\alpha$-approximation theorem for $Q^{\infty}$-manifolds, Topology Appl. 12 (1981), $289-304$.

11. __ An unknotting theorem in $Q^{\infty}$-manifolds, Proc. Amer. Math. Soc. 82 (1981), $125-132$.

12.

13. Peter L. Renz, The contractibility of the homeomorphism group of some product spaces by Wong's method, Math. Scand. 28 (1971), 182-188.

Department of Mathematics, Vanderbilt University, NashVille, TenNesSEE 37235

Current address: Department of Mathematics, University of Alabama, University, Alabama 35486 